\title{
Sequence Planning for Selective Disassembly Aiming at Reducing Energy Consumption Using a Constraints Relation Graph and Improved Ant Colony Optimization Algorithm
}

\author{
Bingtao Hu ${ }^{(\mathbb{B})}$, Yixiong Feng * (D), Hao Zheng and Jianrong Tan \\ State Key Laboratory of Fluid Power and Mechatronic Systems, Zhejiang University, Hangzhou 310027, China; \\ hubingtao@zju.edu.cn (B.H.); haozheng@zju.edu.cn (H.Z.); egi@zju.edu.cn (J.T.) \\ * Correspondence: fyxtv@zju.edu.cn; Tel.: +86-186-6821-1935
}

Received: 8 July 2018; Accepted: 10 August 2018; Published: 13 August 2018

check for updates

\begin{abstract}
With environmental pollution and the shortage of resources becoming increasingly serious, the disassembly of certain component in mechanical products for reuse and recycling has received more attention. However, how to model a complex mechanical product accurately and simply, and minimize the number of components involved in the disassembly process remain unsolved problems. The identification of subassembly can reduce energy consumption, but the process is recursive and may change the number of components to be disassembled. In this paper, a method aiming at reducing the energy consumption based on the constraints relation graph (CRG) and the improved ant colony optimization algorithm (IACO) is proposed to find the optimal disassembly sequence. Using the CRG, the subassembly is identified and the number of components that need to be disassembled is minimized. Subsequently, the optimal disassembly sequence can be planned using IACO where a new pheromone factor is proposed to improve the convergence performance of the ant colony algorithm. Furthermore, a case study is presented to illustrate the effectiveness of the proposed method.
\end{abstract}

Keywords: energy consumption; selective disassembly; disassembly sequence planning; constraints relation graph; improved ant colony optimization algorithm

\section{Introduction}

While rapid technological development has brought unprecedented economic prosperity, human society is now facing huge pressures from environmental damage and resource shortage. As a sign of social progress, large-scale industrialization now stands accused of polluting the environment, producing waste and threatening human health. Instead of pure economic growth, sustainable development is now more popular among people all over the world. Thus, the recycling and remanufacture of mechanical equipment have become priorities to be considered by industry [1-4]. Recently, researchers have started to focus on design and decision-making for recycling [5-9]. Disassembly, as a key step of recycling and remanufacturing, has also attracted wide attention. There are many different studies on theories and methods for disassembly. In general, these studies all focus on three essential problems: disassembly modeling, disassembly sequence planning and disassembly evaluation [10].

Disassembly modeling is about how to represent the mechanical product, including its components and the position and constraint relationships between each other. Aydemir-Karadag et al. worked on disassembly line balancing problem (DLBP) and optimized the line balance and design costs objectives by using AND/OR graphs [11]. Petri Net (PN) is another kind of graphic modeling 
method [12]. A disassembly Petri net (DPN) is proposed in [13] to model the resources constraints and to maximize disassembly profit with scatter search algorithm. Gao et al. developed Petri net and presented a fuzzy reasoning Petri net (FRPN) model to solve the problem about rules containing negative literals addressed in PN model [14]. Li et al. represented disassembly constraint graph (DCG) as a prototype system to solve the object-oriented disassembly problem [15]. Disassembly feasibility information graph (DFIG) is used in [16] to describe the product disassembly sequence and operation information and solve disassembly sequence planning problem. Luo et al. introduced an integrated multi-layer model to enable a very efficient search for the disassembly sequence [17]. Although different kinds of disassembly models have been proposed, most of them require a detailed review of all the components and the constraints between each other. When the structure of the mechanical equipment becomes complicated, this process can be very confusing. Besides, it is also unnecessary for selective disassembly.

Disassembly evaluation is to find the best disassembly sequence according to a specific standard. The evaluation criteria have a lot to do with the purpose of the disassembly. For example, sequence planning aiming at maximum disassembly efficiency may demand fewer changes of disassembly tools to save time, but target-oriented disassembly may sacrifice efficiency for the completeness of the certain component. Thus, when the purpose of the disassembly changes, the feasible sequences for the same mechanical product change accordingly.

As the number of components to be disassembled gets large, the search space of feasible solution will become incredibly wide, which makes traditional methods lose effectiveness but heuristic algorithms become very useful. Ilgin et al. proposed a genetic algorithm (GA)-based simulation optimization approach to determine the disassembly sequence [18]. Go et al. studied automotive component reuse through end-of-life stage of mechanical products and used GA to solve the fitness function which is dependent on the increment in disassembly time [19]. Hui et al. provided a genetic algorithm to find out feasible disassembly solutions efficiently [16]. Optimal or near-optimal solutions were obtained by GA in [20] for disassembly line balancing problems. Wang et al. presented an intelligent selective disassembly approach based on ant colony optimization (ACO) algorithm to minimize the reorientation of assemblies and removal of components [21]. A novel multi-objective ant colony optimization (MOACO) algorithm is proposed in [22] to solve the multi-objective disassembly line balancing problem. Shan et al. used ACO in the disassembly sequence planning problem considering the component number, disassembly tools and disassembly direction [23]. Li et al. developed a Particle Swarm Optimization (PSO)-based selective disassembly planning method to support remanufacturing and recycling of waste electrical and electronic equipment (WEEE) [24]. A new approach based on PSO with a neighborhood-based mutation operator was proposed in [25] to solve sequence-dependent disassembly line balancing problem (SDDLBP). The artificial bee colony $(\mathrm{ABC})$ algorithm was used to make decisions regarding energy-savings for DLBP [26], and its fuzzy extension, the hybrid discrete $A B C$ algorithm, was proposed to deal with the uncertainty of the real-world disassembly systems [27].

Although researchers have proposed a variety of models and algorithms to resolve disassembly issues, some problems remain unsolved in terms of recycling. Most work is about complete disassembly, which means all the components of the mechanical product need to be disassembled, but the research about selective disassembly is much seldom performed. Some research relies on assembly or design experience [28-30], but these experience may be hard to obtain. Some research based on the idea of modularization focuses on the identification of subassembly for given mechanical product but only applies to products with a high degree of modularity or a small number of components. Besides, it is still a complete disassembly problem after the identification of subassembly. The problem about target-oriented disassembly for recycling is more complicated:

(1) The mechanical product contains a lot of components with confusing coupling relationship which requires identification of subassembly; 
(2) The number of the components of the subassembly is still variable when planning the disassembly sequence;

(3) Evaluation criteria are diverse so that proper optimization method needs to be conducted.

In response to the issues above, a hybrid optimization method aiming at reducing energy consumption based on constraints relation graph (CRG) and improved ant colony optimization algorithm (IACO) is proposed in this paper. The main contributions of this research are summarized as follows:

(1) The CRG is first proposed to model the mechanical product. The identification of the subassembly whose center is the target component through CRG and the pruning method can both reduce the search space of feasible disassembly sequences;

(2) Compared to the existing research about disassembly sequence planning, we have established elaborate models considering different kinds of energy consumption for more realistic situations aiming at energy saving;

(3) This paper demonstrates the potential of ACO in dealing with the dynamic change of the number of the components when planning the sequence for a selective disassembly. Furthermore, we have proposed an improved ant colony algorithm to promote efficiency. The comparison between the results of the IACO, ACO and other heuristic algorithm are detailed presented.

The rest of this paper is organized as follows: in Section 2, a detailed description of CRG and IACO is provided. First the concept and pruning method of CRG are illustrated. Then the IACO is used to plan the optimal disassembly sequence. All the key parts of the algorithm, including optimization strategy and evaluation objective, are proposed. Section 3 describes a complete case study. The results using IACO and ACO are compared to illustrate the effectiveness of the proposed method. Finally, some conclusions are given in Section 4.

\section{Preliminaries and Problem Statement}

\subsection{Basic Notions and Pruning Method of CRG Model}

\subsubsection{Basic Motions of the CRG}

There are a lot of constraints inside a complex mechanical product. To clarify the relationship between these constraints, a proper model needs to be established. Thus the CRG, which is designed to delineate the constraint information of the product, is proposed.

There are two different types of constraints between the components inside a mechanical product. Type A of the constraints is formed by position or shape of the components. For example, a bearing is constrained to a shaft by shape and can only be removed along the axial direction. There is no precedence relationship between the related components. It is usually not necessary to employ disassembly tools to release this type of constraints and the disassembly operation does not cause damage to the components. Type B of constraints is formed by connecting parts and needs disassembly tools to get released (Figure 1a) or will cause damage to the related components (Figure 1b). Since the connecting parts need to be removed before releasing constraints, there is precedence relationship between the related components. In the CRG model, undirected and directed edges are used to represent the two different types of constraints, respectively.

The CRG model is a hybrid graph to represent and constraint information between the components. Basically, a CRG can be defined as follows:

$$
G=\{V, E, D, W, R\}
$$

where $G$ is a graph whose center is the target component and denotes the subassembly containing the target component. $V=\left\{v_{i} \mid i \in m_{G}\right\}$, is the point set of graph $G$, where $m_{G}$ is the number of vertices 
in the graph. Point $v_{i}$ is a node and denotes a component that can not be further disassembled. $E$ is the undirected edge set. Every element $e_{i, j}$ in it denotes a Type-A constraint between the two related vertices. $D$ is the directed edge set. Every element $d_{i, j}$ in it denotes a Type-B constraint between the two related vertices. The directed edge in Figure 2a pointing from node 3 to node 4 means that component 3 must be disassembled before component 4 . In the disassembly process, edges represent the disassembly operations that release the constraint. When an edge is removed from a graph, the operation it represents is carried out. $W$ is the weight set. $w_{i, j}$ is some kind of weight loaded on related edges. $R$ indicates the AND/OR relationship. One component may take a few constraints from different components and the constraints need to be released in proper order, which is called the AND relationship. AND relationship does not need to be underlined in a graph, but sometimes these constraints do not have priority over each other and can be removed at the same time, which is the OR relationship. The OR relationship should be marked in a graph.

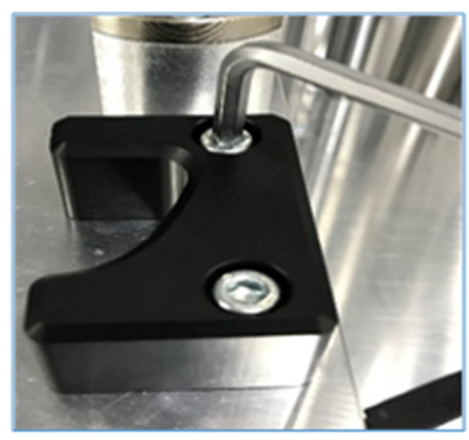

(a)
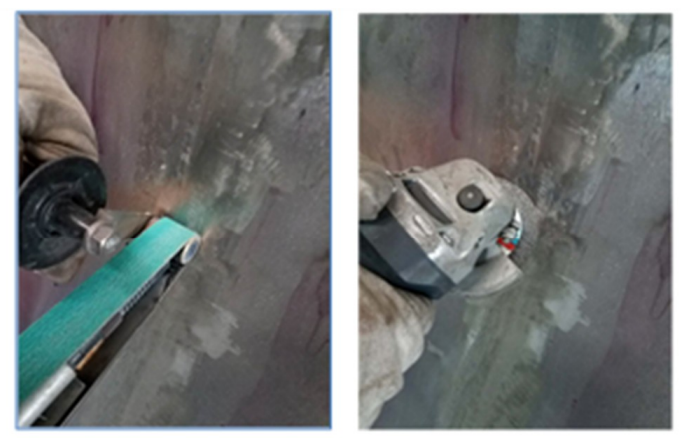

(b)

Figure 1. Disassembly operation with tools or causing damage to components. (a) Use a screw driver to release the screw connection; (b) Heat the components and scrape the solder with a scraper.

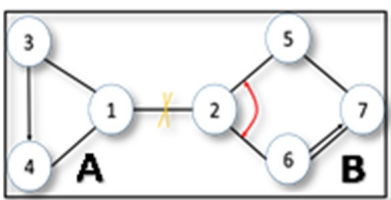

(a)

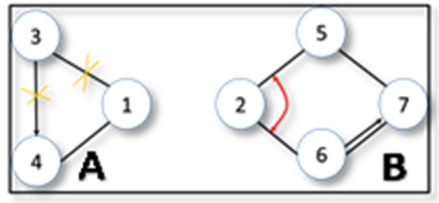

(b)

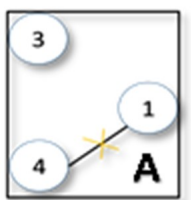

(c)

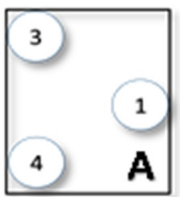

(d)

Figure 2. Prune the CRG to obtain the target component. (a) The undirected edge between node 1 and node 2 can be removed; (b) Remove the aforementioned undirected edge and another two edges can be removed in subassembly A; (c) Repeat the pruning method; (d) The component 1 is now free.

A mechanical product can be represented by a CRG and the CRG needs to be pruned to get the subassembly. The pruning process can separate the target component from the complex product. Some special points are very useful to promote the pruning process.

A free node denotes a component that can be directly disassembled from the product. In the graph, it is a vertex with no directed edge pointing to and no more than one undirected edge. The node 3 in Figure 2 is a free node. It can be used as the starting point in a disassembly sequence. When a point becomes free after disassembly operation, it can be recorded into the disassembly sequence. The aim of the sequence planning is to make the target-component node free so that the target component can be removed.

A cut node is a node whose removal will make the graph lose its connection [31]. Node 1 and node 2 in Figure 2 are both cut nodes. The cut node is very useful in disassembly sequence planning because the easiest way to get a subgraph is to release the connection between cut nodes. Correspondingly the subassemblies represented by the subgraphs can be obtained. 


\subsubsection{Pruning Method of the CRG}

The CRG provides a way to switch the disassembly object from the complex mechanical product to a simple graph. Since the edges in the CRG represent the constraints between the components and also the corresponding disassembly operations to release them, the pruning of the graph can represent the disassembly of the mechanical product. By pruning the CRG, the number of the components that need to be disassembled is decreased so that the search space of the feasible disassembly sequence is compressed.

As shown in Figure 2a, node 1 represents the target component to be recycled and needs to be detached from the graph. Firstly, the cut nodes are located to identify the subassembly. In this case, node 1 itself and node 2 are both cut nodes. There is an undirected edge between them which means some kind of constraint. Once the edge is removed, the graph is divided into two subgraphs (Figure 2b). Since the target node 1 is in subassembly A, the subassembly B can be simply put aside.

Secondly, a free node is supposed to be found in A as the starting point of the disassembly sequence. For node 1, node 3 and node 4 in subgraph A, only node 3 is a free node. So node 3 and its related edges can be removed and then node 4 becomes a free node. Repeat the step above until node 1 becomes free. In the case shown in Figure 2, the disassembly sequence is $3 \rightarrow 4 \rightarrow 1$.

If the target component is represented by node 2, the situation is a bit different because there seems to be no free node in B. In the real case, the product needs to be rechecked to see if some constraints can be released properly to get a free node, but in this case, the relationship line $R$ (red line in subgraph B) can help save the trouble. The relationship line $R$ indicates that two constraints can be released at one time, which means technically node 2 is a free node.

It should be noticed that under some circumstances, the absence of free nodes indicates that the target component cannot be obtained without damage to others. The disassembly operations for highly modular subgraph may be performed with high cost. In other words, the benefits brought by recycling may be offset by the cost of disassembly.

\subsection{Planning and Evaluation for the Disassembly Sequence}

\subsubsection{Disassembly Sequence Evaluation}

Once a free node is identified, pruning the CRG indicates that the related disassembly operations are carried out. Many different sequences can be feasible to get the target component but disassembly operations performed in different sequences will be at different costs. Among all these feasible sequences, the one at minimum cost needs to be found. In general, the cost can be evaluated by the following factors [16-19,32,33]:

(1) Basic operation-preparing time;

(2) Time of disassembly tools change;

(3) Time of disassembly operation direction change, with tools or not;

(4) Time, economic or energy costs caused by disassembly operations.

From an energy perspective, all the factors can increase the total energy consumption of the disassembly process.

The basic operation-preparing time affects the basic energy consumption caused by clamping equipment, including equipment starting and stopping, equipment idling and auxiliary (such as coolant and lighting). So the total basic equipment energy consumption can be expressed as:

$$
E_{b}=\sum_{i=1}^{M} P_{b i} \cdot t_{b i}
$$

where $P_{b i}$ is the average power, $t_{b i}$ is the toal preparing time of the $i$-th equipment and $M$ is the number of equipment. 
To accomplish the disassembly process, the disassembly tool may change according to the types of connection between every two adjacent components. Since each time the tool changes will cause energy consumption, the total energy consumption of tool changes of the disassembly process can be expressed as:

$$
E_{c}=\sum_{i=1}^{T_{c}} e_{c i}
$$

where $e_{c i}$ is the energy consumption of the $i$-th tool change and $T_{\mathcal{c}}$ is the time of tool changes.

Similarly, disassembly operations often need to be performed from different directions, which may require the components to be repositioned. The total energy consumption caused by disassembly operation direction changes can be expressed as:

$$
E_{d}=\sum_{i=1}^{T_{d}} e_{d i}
$$

where $e_{d i}$ is the energy consumption of the $i$-th disassembly operation direction change and $T_{d}$ is the time of disassembly operation direction changes.

$E_{b}, E_{c}$ and $E_{d}$ are all about the energy consumed by support work $E_{s}$. The largest part of energy consumption during the disassembly process is caused by disassembly operations which include releasing the inter-connection constraints and removing the free component. The research in [34] shows that the physical energy which is required for release of an inter-connection is determined by the type of connection. In [35], Zhang quantifies the relative impact of the type of connection on the energy consumption in the disassembly process considering manufacturing process, material, recovery level and assembly/disassembly performance. The total energy consumption caused by releasing the inter-connections is expressed as:

$$
E_{r}=\sum_{i=1}^{T_{r}} e_{r i}
$$

where $e_{r i}$ is the energy consumption of releasing the $i$-th inter-connection and $T_{r}$ is the number of releasing operations.

The total energy consumption caused by component-removing operations is expressed as:

$$
E_{m}=\sum_{i=1}^{T_{m}} e_{m i}
$$

where $e_{m i}$ is the energy consumption of the $i$-th component-removing operation and $T_{m}$ is the number of component-removing operations. In the CRG, the weight $w_{i, j}$ loaded in the edge connecting nodes $i$ and $j$ represents the energy consumption when releasing the inner-connection between the components and removing the disassembled component. $w_{i, j}$ is determined by connection type and the disassembly operations, that is, $w_{i, j}$ is related to the corresponding $e_{r i}$ and $e_{m i}$.

Based on the foregoing analysis about the energy consumption, the total energy consumption required to complete the disassembly process can be calculated as:

$$
\text { Energy }=\underbrace{E_{b}+E_{c}+E_{d}}_{E_{s}}+\underbrace{E_{r}+E_{m}}_{W}
$$

To minimize Energy, the corresponding parameters $t_{b i}, T_{c}, T_{d}, T_{r}$ and $T_{m}$ are expected to be as small as possible. But it should be noted that $e_{c i}, e_{d i}, e_{r i}$ and $e_{m i}$ are not constants, they are all variable according to the disassembly sequence. For example, two successive disassembly operations using the same tool in the same disassembly direction consume less energy than that using different tools in different disassembly directions. 
When the number of components to be disassembled gets large, the solution space of feasible disassembly sequences becomes incredibly wide and calculating the energy consumption of each feasible sequence to find the optimal one becomes very complicated. Heuristic algorithms have a lot of advantages in this issue, so in this paper the IACO is used to solve this problem.

\subsubsection{Disassembly Sequence Planning}

The sequence planning for a target-oriented disassembly process is an optimization problem with a variable number of components. ACO is an efficient artificial intelligence procedure that imitates the behavior of an ant colony finding the shortest path between the nest and the source of food. Each ant leaves volatile secretion (pheromone) on the path it passed; then the following ants will either repeat the path or find a new one. The more the pheromone is left on the path, the more attractive the path becomes to the following ants. The amount of pheromone on the paths with less or no ants repeating will decrease because the pheromone volatiles at a certain rate. Therefore, the shortest path will be visited most with the continuous action of the colony.

In the ACO algorithm, the total pheromone $Q$ released by each ant in the entire route is a constant. In this paper, a new pheromone factor is proposed to improve the performance of the algorithm because it can adapt to the variable number of the components to be disassembled. That is, the smaller the number of components to be disassembled is, the larger the pheromone factor becomes. The experiment results show that this method can speed up the convergence of the algorithm.

To combine IACO and CRG to determine the minimum energy consumption, some issues have to be addressed:

(1) For the disassembly problem, each ant's path represents a disassembly sequence and the nodes on the path represent disassembled components. Due to the constraints between the components, the ants are supposed to follow certain rules when passing through these nodes. The matrices of undirected edge (UM) and directed edge (DM) are adopted in this paper to reflect the constraint relationship between the components.

(2) When an ant moves from node $i$ to node $j$, the constraints between the components represented by these two nodes are released. The distance traveled by the ant represents the energy consumption caused by the disassembly operation.

(3) For a complete disassembly problem, the end of the algorithm is that all the components are disassembled. But for a selective disassembly problem, the algorithm comes to an end when the target component is disassembled.

Then the optimal sequence can be obtained using IACO. The detailed description for each step of the proposed algorithm are as follows:

(1) Initialization. The number of ants in colony is $m$ and the number of components to be disassembled is $n$ ( $m$ is constant and $n$ is variable). In the disassembly process, the total energy consumption removing component $j$ from its adjacent component $i$ is $e c_{i, j}$. At time $t$, the pheromone concentration on the path between the nodes is $\tau_{i, j}(t)$. At the initial moment, $\tau_{i, j}(0)$ is the same everywhere and is set $\tau_{i, j}(0)=0$.

(2) Sequence planning. To apply the IACO to plan the disassembly sequence, the subassembly identified by pruning the CRG must be converted into numerical data form using constraint matrices. There are two kinds of constraint matrices, which are obtained according to the information of directed and undirected edges in the CRG, respectively. The constraint matrices represent the constraint relationship between parts in Boolean form. In the matrix of undirected edge (UM), each element is defined as follows:

$$
u m_{i, j}=\left\{\begin{array}{ll}
1, & \text { if there are an undireted edge } \\
\text { between components } i \text { and } j \\
0, & \text { otherwise }
\end{array} \quad\{i, j \in C R G\}\right.
$$


$\mathrm{UM}$ is a symmetric matrix and every diagonal element is zero. In the matrix of directed edge (DM), each element is defined as follows:

$$
d m_{i, j}=\left\{\begin{array}{ll}
1, & \text { if there are an undireted edge } \\
\text { between components } i \text { and } j \\
0, & \text { otherwise }
\end{array} \quad\{i, j \in C R G\}\right.
$$

The same as UM, each diagonal element is zero. The component $i$ whose constraint matrices satisfy the following conditions can be identified as a free node:

(1) In the UM, there is no more than one " 1 " in the row $u m_{i}$;

(2) In the DM, there is no " 1 " in the row $d m_{i}$.

When the component represented by a free node $i$ is disassembled, every element of the $i$-th row and the $i$-th column in both UM and DM becomes " 0 " and new free nodes appear. Repeat the process until the target point becomes a free node, which means the target component can be disassembled from the product.

Every free node found by pruning the initial CRG can be settled as the starting point of the IACO. The starting point is also the beginning of the disassembly sequence. When an ant visited point $i$, the related constraints are released and the energy consumption is recorded. Then some new free nodes appear and are put into the point set $P_{k}$, in which all the points are allowed to visit. The ordered point set $P_{S}$ records the points that the ant has visited as the disassembly sequence. Ants will decide the node to visit next according to the pheromone concentration. The probability that the ant $k$ will transfer from point $i$ to point $j$ at time $t$ is:

$$
P_{i, j}^{k}= \begin{cases}\frac{\left[\tau_{i, j}(t)\right]^{\alpha} \cdot\left[\eta_{i, j}(t)\right]^{\beta}}{\sum_{S \in P_{k}}\left[\tau_{i, s}(t)\right]^{\alpha} \cdot\left[\eta_{i, s}(t)\right]^{\beta}}, & S \in P_{k} \\ 0, & S \notin P\end{cases}
$$

where $\eta_{i, j}(t)=1 / e c_{i, j}$ is the heuristic function representing the expectation degree to which the ants are moving from point $i$ to point $j ; \alpha$ is the importance degree factor of pheromone; the bigger it is, the greater the pheromone will impact the transfer process; $\beta$ is the importance degree factor of heuristic function; the bigger it is, the greater the heuristic function will impact the transfer process. Once the target point is visited by the ant $k$, the disassembly process finishes and the total energy consumption $E_{k}$ will be output according to point set $P_{s}$. Then the $(k+1)$-th ant will repeat the process and that total energy consumption $E_{k+1}$ is obtained. Finally, the minimum value in the energy consumption vector $E_{v}$ consisting of $E_{k}(\forall k \in[1, m])$ is found and the corresponding disassembly sequence is the result of this generation. The result will be used to adjust the pheromones concentration of the next generation and guide the ants to plan their paths.

(3) Pheromone updating. While the ants are releasing pheromone, the pheromones between the nodes are gradually volatilizing. After the whole generation has finished the searching process, the pheromones need to be updated as follows:

$$
\left\{\begin{array}{c}
\tau_{i, j}(t+1)=(1-\rho) \cdot \tau_{i, j}(t)+\Delta \tau_{i, j} \\
\Delta \tau_{i, j}=\sum_{k=1}^{m} \Delta \tau_{i, j}^{k}
\end{array}\right.
$$

where $\rho$ is the volatilization factor; the bigger it is, the faster the pheromones volatilize; $\Delta \tau_{i, j}^{k}$ is the amount of pheromone that the $k$-th ant left between node $i$ and node $j$, and $\Delta \tau_{i, j}$ is the sum amount of pheromones that all the ants left between the two nodes. In this paper, $\Delta \tau_{i, j}^{k}$ is calculated as:

$$
\Delta \tau_{i, j}^{k}= \begin{cases}\frac{Q \cdot e^{\frac{n_{d}}{n_{k}}}}{E_{k}}, & \text { if the } k-\text { th ant moved from } i \text { to } j \\ 0, & \text { otherwise }\end{cases}
$$


where $e^{\frac{n_{d}}{n_{k}}}$ is the pheromone factor. $n_{d}$ indicates the number of the components included in the subassembly and is a constant, $n_{k}$ indicates the number of components in the disassembly sequence represented by the $k$-th ant's path and can be variable. Obviously we have:

$$
n_{k} \leq n_{d}
$$

When $n_{k}=n_{d}, \forall k \in\{1,2, \ldots, m\}$, the $Q \cdot e^{\frac{n_{d}}{n_{k}}}$ remains a constant which indicates that this is a complete disassembly problem. When $n_{k}<n_{d}, \exists k \in\{1,2, \ldots, m\}$, it becomes a selective disassembly problem and the $k$-th ant leaves more pheromone on the path than others, that is, the $k$-th ant's path is more attractive to the following ants.

Repeat the above process and the best disassembly sequence containing the target component aiming at lowest energy consumption will be found. The architecture of the proposed method combining CRG and IACO is summarized in Figure 3. The content inside the red dotted line is the process about establishment and pruning of the CRG and the content inside the blue dotted line is the process about using the IACO to get the best disassembly sequence.

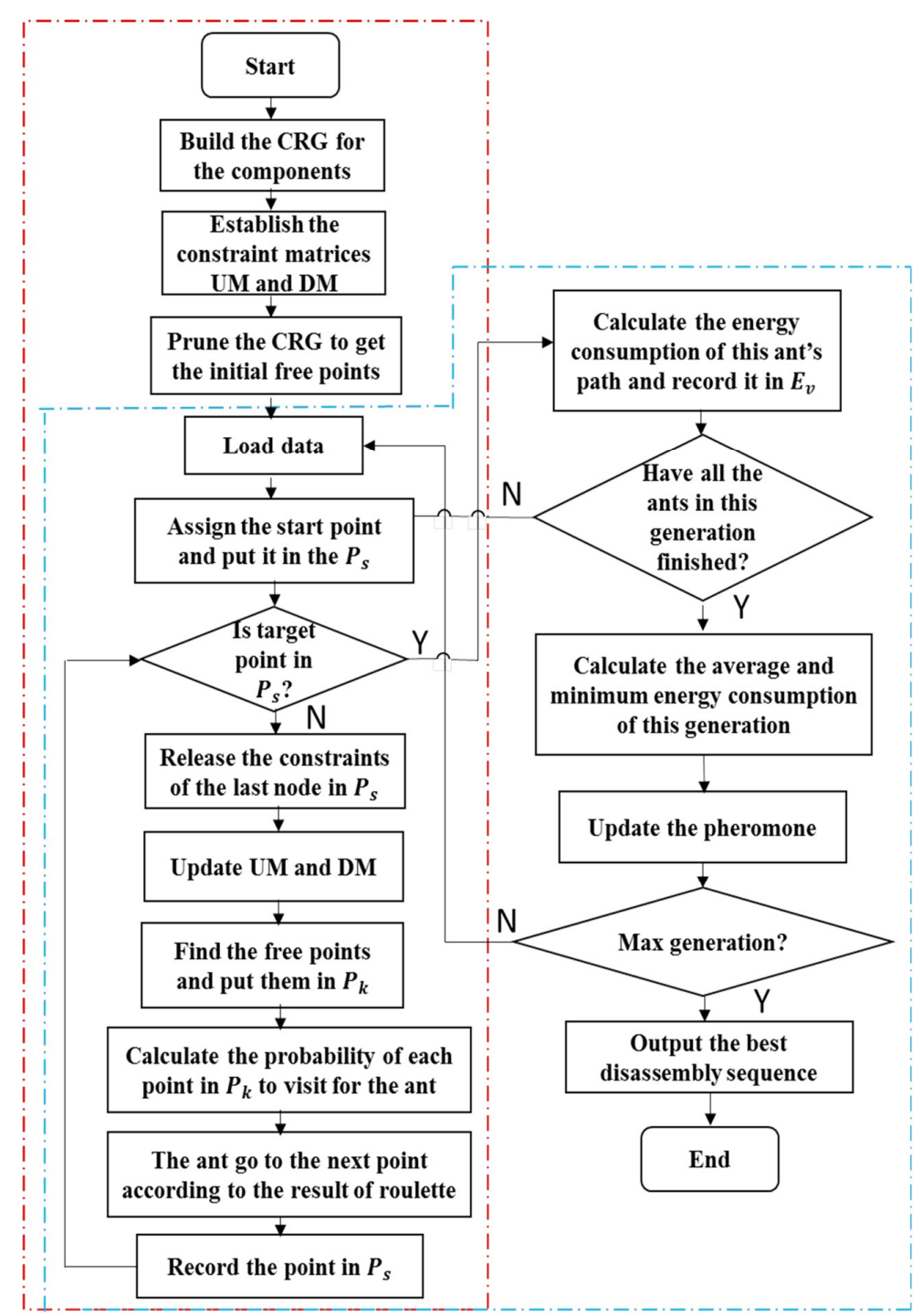

Figure 3. The architecture of the proposed method combining CRG and IACO. 


\section{Case Study and Discussion}

\subsection{Case Study}

The proposed method has been applied to a rolling machine for the recycling of a target critical component (the component in light green in Figure 4 represents the target component). The whole machine contains more than 300 components, which makes it hard and unnecessary for a complete disassembly. To reduce the disassembly cost and lower the energy consumption, the CRG model is built and the best disassembly sequence is determined through IACO. The main steps to recycle the target component in this case are as follows:

(1) Build the CRG model.

For the ease of illustration, the target component and its associated components are numbered in Figure 5. The disassembly tool and operation direction are listed in Table 1. Take the target component as the center and analyze the constraints relationship of its surrounding components, then draw the first step of CRG. Based on this, the second step is carried on and the free nodes are found (Figure $6 a, b$ ). The process is repeated until the subassembly containing the target component is identified (Figure 6c-e). Finally, a subassembly with 14 components which is much simpler than the original machine is obtained. Its boundary points are all free nodes, that is, the disassembly can be removed from the product as an entirety.

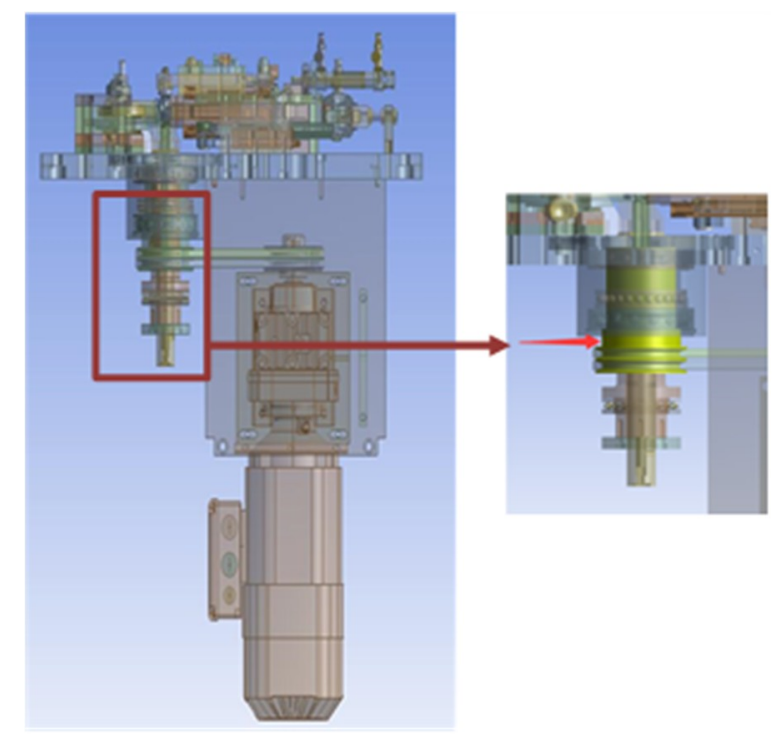

Figure 4. The target component.

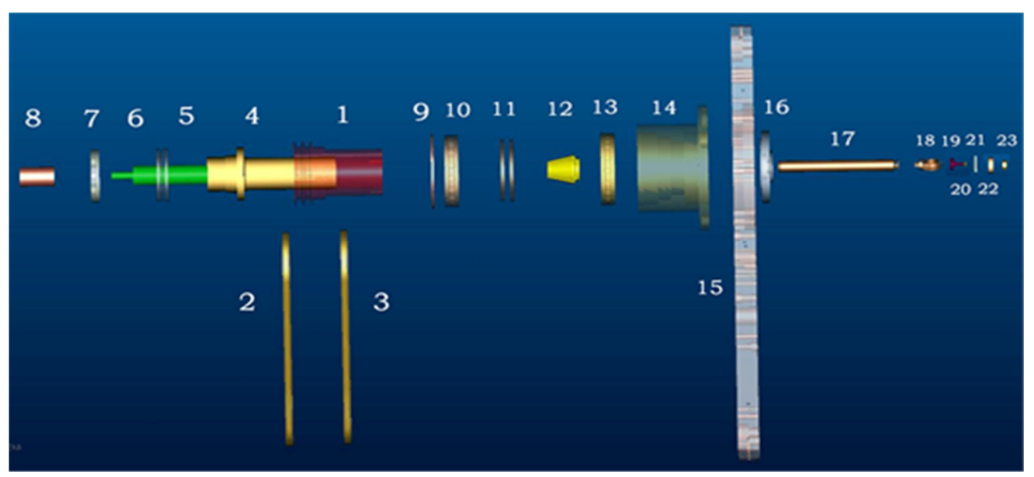

Figure 5. The target component and its associated components. 
Table 1. The disassembly tool and operation direction of the components.

\begin{tabular}{|c|c|c|c|c|c|c|c|}
\hline Number & Component & Tool & Direction & Number & Component & Tool & Direction \\
\hline 1 & Drive shaft & Clamp & $-x$ & 13 & Bearing & Hand & $-x$ \\
\hline 2 & Belt & Belt Spanner & $+y /-y$ & 14 & Shaft & Screwdriver & $+x$ \\
\hline 3 & Belt & Belt Spanner & $+y /-y$ & 15 & Plate & Carrier & $+x /-x$ \\
\hline 4 & Shaft & Clamp & $-x$ & 16 & Disc & Screwdriver & $+x$ \\
\hline 5 & Shaft & Hand & $-x$ & 17 & Tube & Clamp & $+x$ \\
\hline 6 & Bearing & Clamp & $-x$ & 18 & Eye & Clamp & $+\mathrm{x}$ \\
\hline 7 & Disc & Screwdriver & $-x$ & 19 & Insert & Tweezers & $+x$ \\
\hline 8 & Shaft & Clamp & $-x$ & 20 & Disc & Tweezers & $+x$ \\
\hline 9 & Gasket & Clamp & $+x$ & 21 & Cover & Clamp & $+x$ \\
\hline 10 & Bearing & Hand & $+x$ & 22 & Disc & Tweezers & $+x$ \\
\hline 11 & Disc & Hand & $+x$ & 23 & Cover & Clamp & $+x$ \\
\hline 12 & Plasma & Pliers & $+x$ & & & & \\
\hline
\end{tabular}

(2) Establish the constraint matrices.

As stated before, the constraint matrices can be obtained from the CRG model. Exclude the unnecessary components from the disassembly sequence and the UM and DM of the remaining components of Figure 6e are presented in Tables 2 and 3, respectively. The omitted elements in the matrices are all " 0 " and mean that there are no constraints between the components.

Table 2. The UM of Figure 6e.

\begin{tabular}{|c|c|c|c|c|c|c|c|c|c|c|c|c|c|c|}
\hline Component & 1 & 4 & 5 & 6 & 7 & 8 & 9 & 10 & 11 & 12 & 13 & 14 & 16 & 17 \\
\hline 1 & & 1 & & & & & & 1 & 1 & & 1 & & 1 & \\
\hline 4 & 1 & & 1 & & 1 & 1 & & & & & & & & \\
\hline 5 & & 1 & & & & & & & & & & & & \\
\hline 6 & & & & & & & & & & & & & & 1 \\
\hline 7 & & 1 & & & & & & & & & & & & \\
\hline 8 & & 1 & & & & & & & & & & & & \\
\hline 9 & & & & & & & & 1 & & & & & & \\
\hline 10 & 1 & & & & & & 1 & & & & & & & \\
\hline 11 & 1 & & & & & & & & & & & & & \\
\hline 12 & & & & & & & & & & & & & & 1 \\
\hline 13 & 1 & & & & & & & & & & & & & \\
\hline 14 & & & & & & & & & & & & & 1 & \\
\hline 16 & 1 & & & & & & & & & & & 1 & & \\
\hline 17 & & & & 1 & & & & & & 1 & & & & \\
\hline
\end{tabular}

Table 3. The DM of Figure 6e.

\begin{tabular}{|c|c|c|c|c|c|c|c|c|c|c|c|c|c|c|}
\hline Component & 1 & 4 & 5 & 6 & 7 & 8 & 9 & 10 & 11 & 12 & 13 & 14 & 16 & 17 \\
\hline 1 & & & & & & & & & & & & & & \\
\hline 4 & 1 & & & 1 & & & & & & 1 & & & & \\
\hline 5 & & & & & & & & & & & & & & \\
\hline 6 & & & & & & & & & & & & & & \\
\hline 7 & & & & & & & & & & & & & & \\
\hline 8 & & & 1 & & 1 & & & & & & & & & \\
\hline 9 & & & & & & & & & & & & & & \\
\hline 10 & & & & & & & & & & & & & & \\
\hline 11 & & & & & & & & 1 & & & & & & \\
\hline 12 & & & & & & & & & & & & & & \\
\hline 13 & & & & & & & & & 1 & & & & & \\
\hline 14 & & & & & & & 1 & & & & 1 & & & \\
\hline 16 & & & & & & & & & & & & & & \\
\hline 17 & & & & & & & & & & & & & & \\
\hline
\end{tabular}


(3) Calculate the energy consumption.

The total energy consumption $e c_{i, j}$ removing component $j$ from its adjacent component $i$ is determined by support work energy consumption and disassembly operation energy consumption. Since the difference in energy consumption caused by tool changes and disassembly operation direction changes is quite slight, the average $e_{c i}$ is set to $160 \mathrm{~J}$ each time and the average $e_{d i}$ is set to $130 \mathrm{~J}$ each time to simplify the calculation. The average power of the equipment basic energy consumption is set to $0.10 \mathrm{~kW}$.

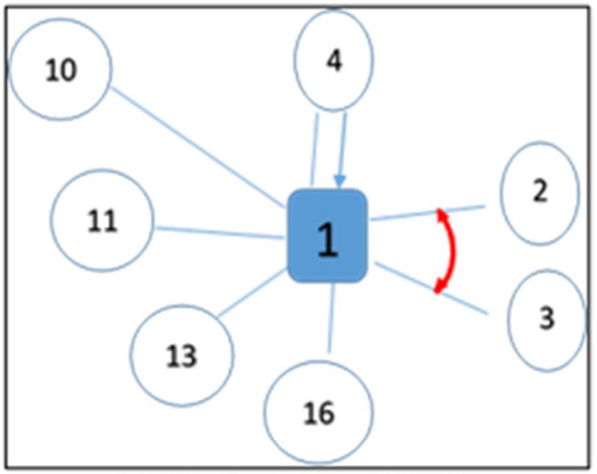

(a) The first step of CRG

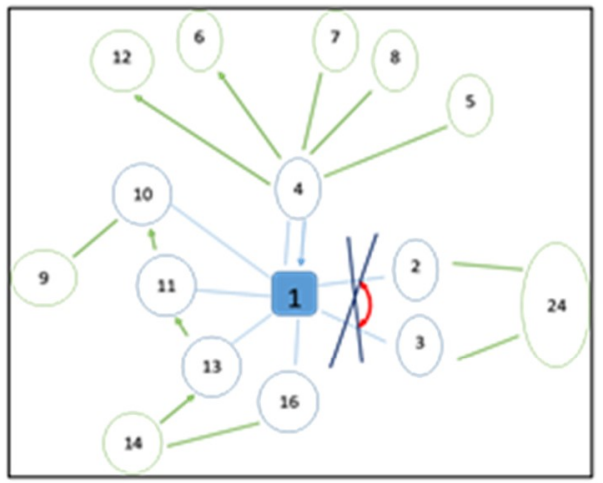

(b) The second step of CRG

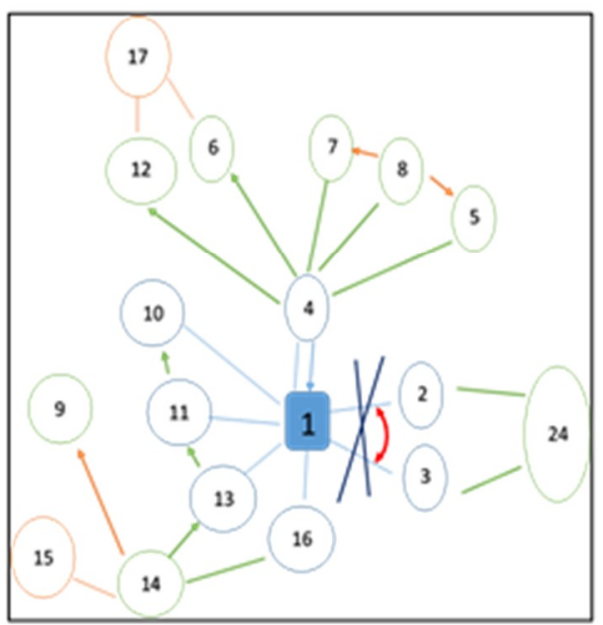

(c) The third step of CRG

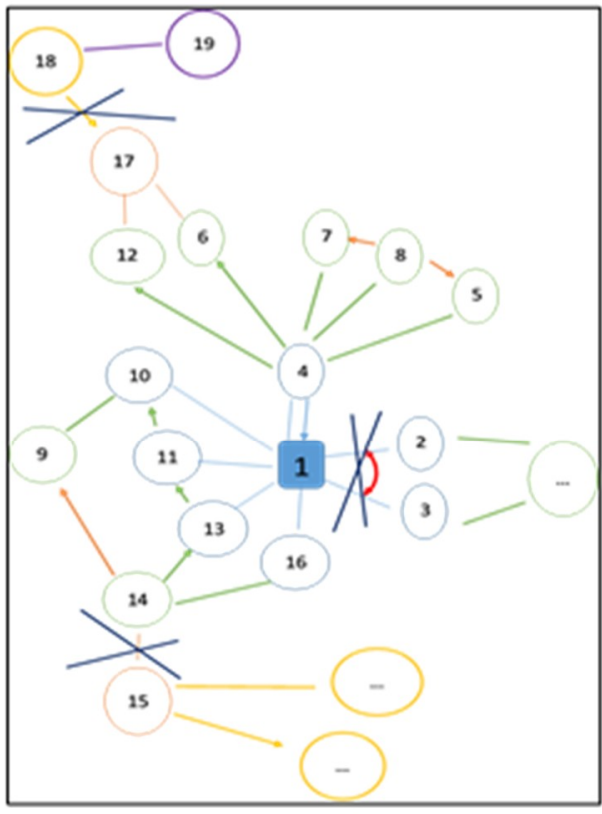

(d) The fourth step of CRG

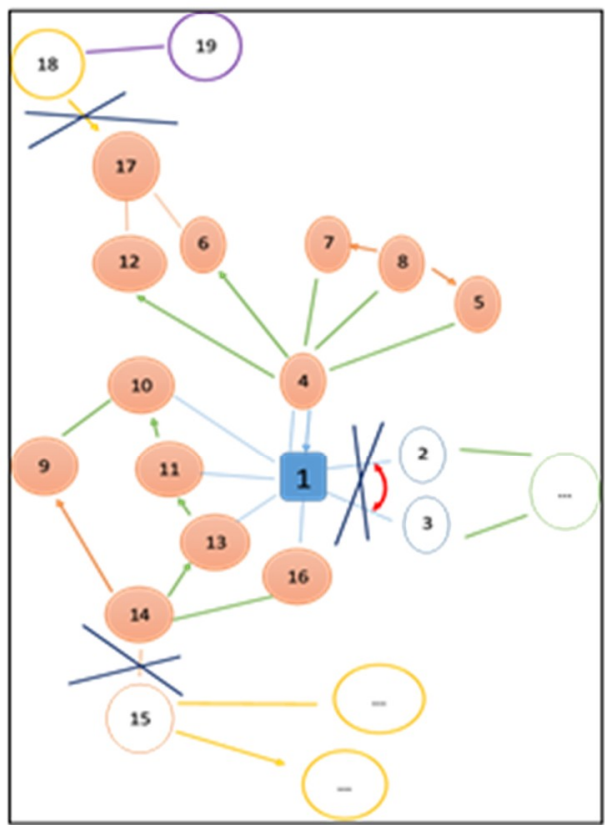

(e) The result of subassembly identification

Figure 6. Build the CRG and identify the subassembly containing target component. 
(4) Disassembly sequence planning using IACO.

In Figure 6e, the free nodes are node 8 and node 14, which can be used as starting points in the disassembly sequence. The typical iterative process using IACO and ACO to get the best disassembly sequence aiming at recycling the target component is shown in Figure 7. Besides, the result of the complete disassembly of the subassembly is also shown. The parameters' values are set as: $\alpha=20, \beta=0.5, \rho=0.05, Q=0.5, m=40, g=160$. The genetic algorithm (GA) is employed for the same case to validate the effectiveness of the proposed method. All the experimental simulations were implemented in MATLAB R2014a and were run on a desktop computer (Inter/Core i7-4790 CPU, $3.60 \mathrm{GHz}$ and $8 \mathrm{~GB}$ RAM with the Windows 10 operating system).

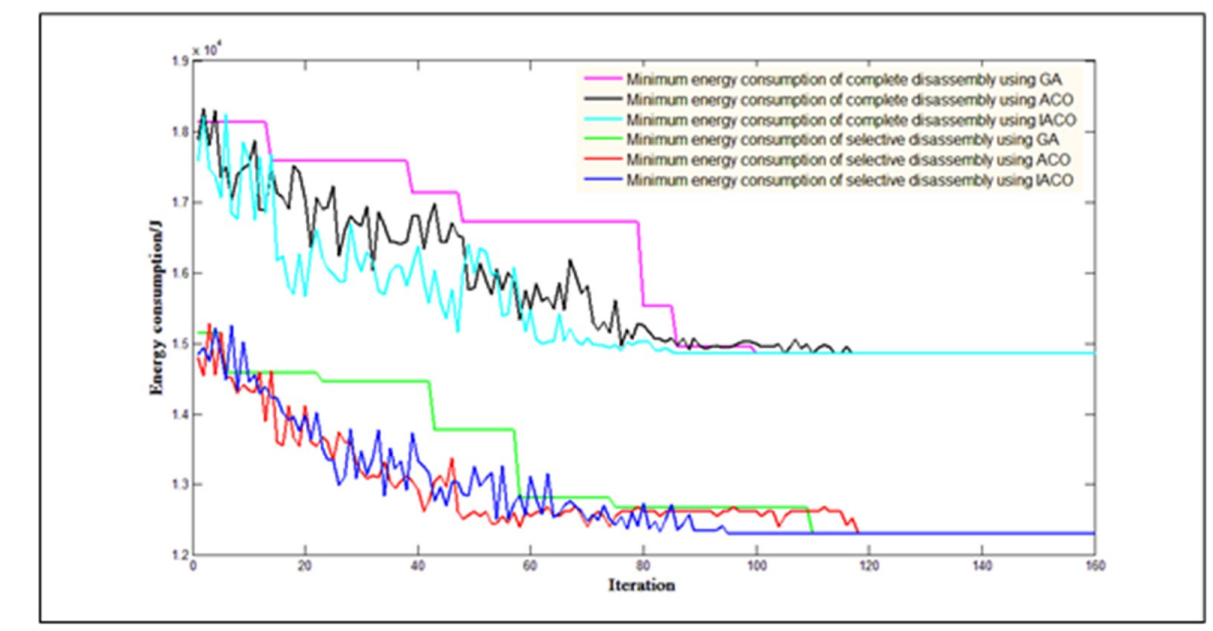

(a) Energy consumption of disassembly sequence with starting point 8 .

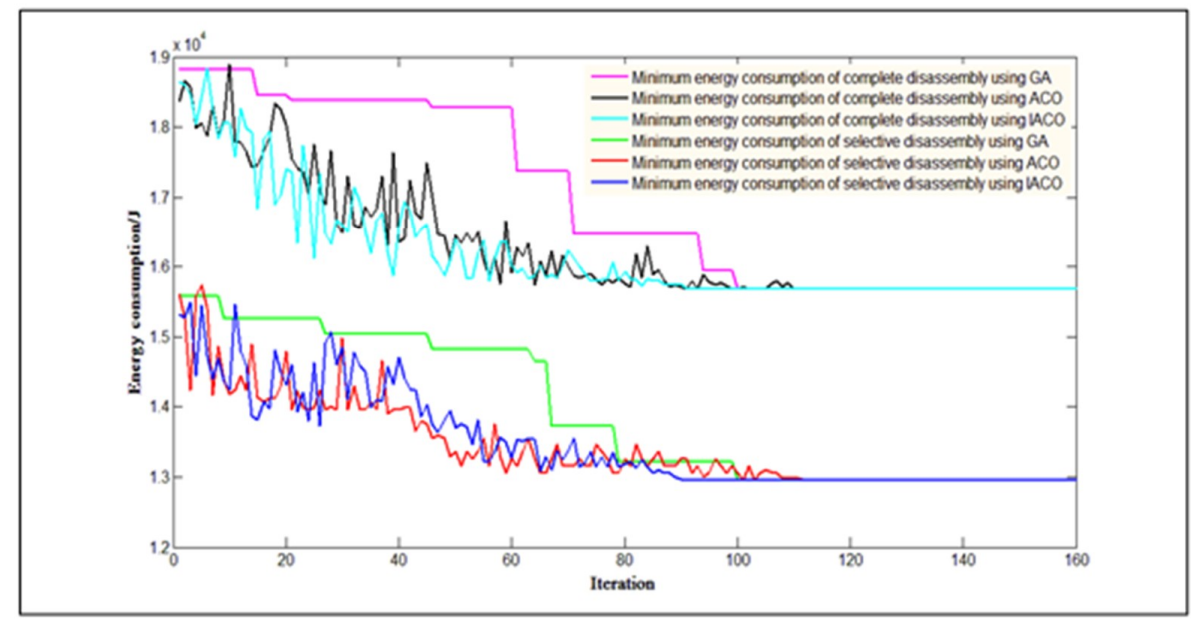

(b) Energy consumption of disassembly sequence with starting point 14.

Figure 7. The iteration process of energy consumption. (a) Energy consumption of disassembly sequence with starting point 8 ; (b) Energy consumption of disassembly sequence with starting point 14 .

\subsection{Discussion}

The result in Figure 7 shows that all the disassembly sequence plans made using GA, ACO and IACO can obtain the best one consuming minimum energy. The detailed comparison is presented in Table 4. Since the results of each run of the heuristic algorithms are not exactly the same, all the approaches are run for 50 times under the same condition to obtain an average. The results indicate that the pheromone factor can accelerate the convergence process. Moreover, GA costs more time than both ACO and IACO because the coding must subject to the inner constraints of the components, which often 
leads to ineffective gene coding after crossover and mutation operations. We also want to make it clear that the traditional GA cannot deal with the changing number of the components, therefore the lengths of the gene chains for complete disassembly and selective disassembly are different.

Table 4. Comparison of the disassembly sequence planning using IACO, ACO and GA.

\begin{tabular}{|c|c|c|c|c|c|}
\hline $\begin{array}{l}\text { Disassembly } \\
\text { Type }\end{array}$ & $\begin{array}{l}\text { Starting } \\
\text { Point }\end{array}$ & Approach & $\begin{array}{c}\text { Time to Obtain the Best } \\
\text { Sequence/s }\end{array}$ & $\begin{array}{l}\text { Iteration to Obtain } \\
\text { the Best Sequence }\end{array}$ & $\begin{array}{l}\text { Efficiency Improvement } \\
\text { for Iteration }\end{array}$ \\
\hline \multirow{5}{*}{$\begin{array}{l}\text { Complete } \\
\text { Disassembly }\end{array}$} & \multirow{3}{*}{8} & IACO & 19.3 & 86 & - \\
\hline & & $\mathrm{ACO}$ & 25.1 & 112 & $23 \%$ \\
\hline & & GA & 87.3 & 104 & $17.3 \%$ \\
\hline & \multirow[b]{2}{*}{14} & IACO & 19.9 & 88 & - \\
\hline & & $\mathrm{ACO}$ & 25.8 & 112 & $21.4 \%$ \\
\hline \multirow{6}{*}{$\begin{array}{l}\text { Selective } \\
\text { Disassembly }\end{array}$} & \multirow{3}{*}{8} & IACO & 18.8 & 84 & - \\
\hline & & $\mathrm{ACO}$ & 23.4 & 107 & $21.5 \%$ \\
\hline & & GA & 70.6 & 99 & $15.2 \%$ \\
\hline & \multirow{3}{*}{14} & IACO & 19.0 & 84 & - \\
\hline & & $\mathrm{ACO}$ & 24.1 & 108 & $22.2 \%$ \\
\hline & & GA & 74.3 & 101 & $16.8 \%$ \\
\hline
\end{tabular}

The energy consumption of the complete disassembly is higher than that of the selective disassembly. Table 5 shows the comparison between them. The subassembly contains 14 components and the selective disassembly requires only 11 of them to be disassembled to obtain the target component. That is, the other three components can be disassembled as an entirety without releasing the inner-connections between them. The selective disassembly saves $2556 \mathrm{~J}$ and $2734 \mathrm{~J}$ energy compared to the complete disassembly with starting point 8 and 14, respectively. The detailed comparison in Figure 8 indicates that the selective disassembly can reduce energy consumption in different aspects of the disassembly process.

Table 5. Comparison of the complete disassembly and selective disassembly.

\begin{tabular}{|c|c|c|c|c|c|}
\hline Disassembly Type & Starting Point & Disassembly Sequence & Tool Change & Direction Change & Energy \\
\hline \multirow{2}{*}{$\begin{array}{l}\text { Complete } \\
\text { Disassembly }\end{array}$} & 8 & $\begin{array}{l}8 \rightarrow 14 \rightarrow 7 \rightarrow 13 \rightarrow 5 \rightarrow 11 \rightarrow 4 \rightarrow 16 \\
\quad \rightarrow 10 \rightarrow 12 \rightarrow 9 \rightarrow 17 \rightarrow 9 \rightarrow 1\end{array}$ & 7 & 6 & 14,855 \\
\hline & 14 & $\begin{aligned} 14 & \rightarrow 8 \rightarrow 7 \rightarrow 13 \rightarrow 5 \rightarrow 11 \rightarrow 4 \rightarrow 16 \\
& \rightarrow 10 \rightarrow 12 \rightarrow 9 \rightarrow 17 \rightarrow 9 \rightarrow 1\end{aligned}$ & 8 & 5 & 15,686 \\
\hline \multirow{2}{*}{$\begin{array}{c}\text { Selective } \\
\text { Disassembly }\end{array}$} & 8 & $\begin{aligned} 8 & \rightarrow 14 \rightarrow 7 \rightarrow 13 \rightarrow 5 \rightarrow 11 \\
& \rightarrow 4 \rightarrow 16 \rightarrow 10 \rightarrow 9 \rightarrow 1\end{aligned}$ & 6 & 6 & 12,299 \\
\hline & 14 & $\begin{array}{l}14 \rightarrow 8 \rightarrow 7 \rightarrow 13 \rightarrow 5 \rightarrow 11 \\
\rightarrow 4 \rightarrow 16 \rightarrow 10 \rightarrow 9 \rightarrow 1\end{array}$ & 7 & 5 & 12,952 \\
\hline
\end{tabular}

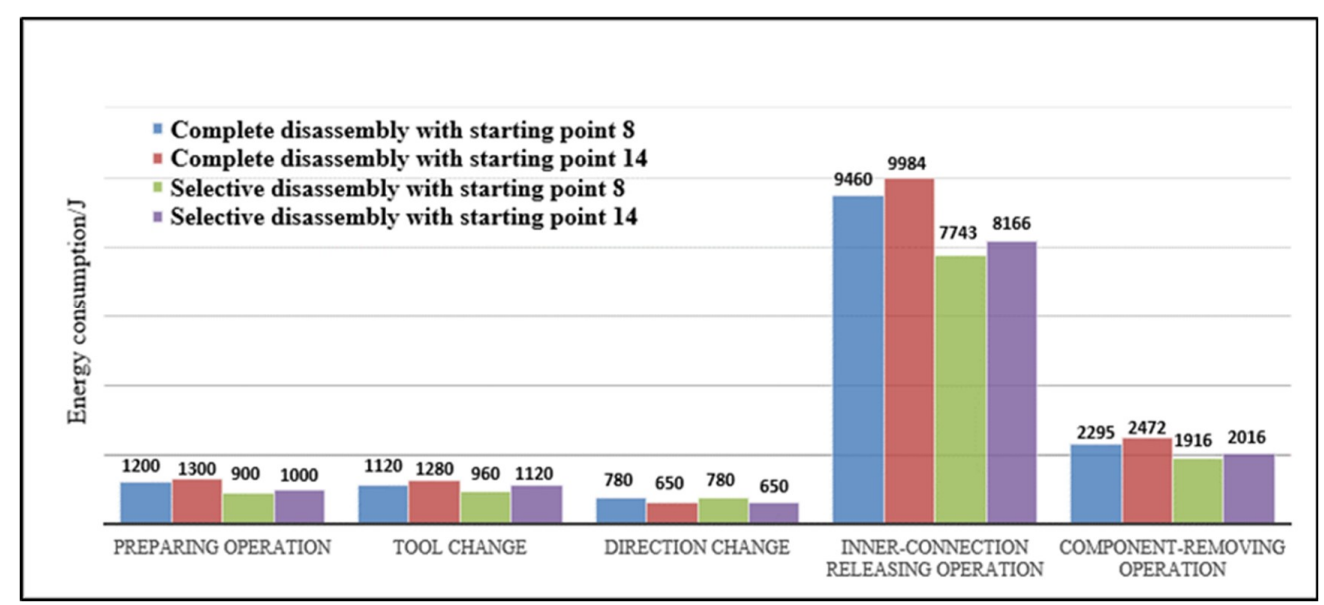

Figure 8. Detailed comparison of different disassembly sequences. 
The disassembly sequences starting with 8 and 14 consume very similar amounts of energy. The difference is caused by sub-sequence $8 \rightarrow 14 \rightarrow 7$ and $14 \rightarrow 8 \rightarrow 7$. It should be noted that sequence planning with different starting points does not necessarily result in sequences containing the same components. For a complex mechanical structure containing a larger number of components, the disassembly sequences with different starting points can be very different.

\section{Conclusions}

To cope with the pressure of resource shortage, more and more researchers want to recycle key components from some end-of-life mechanical products. However, the selective disassembly for the target component remains an unsolved problem. To plan the disassembly sequence and reduce the energy consumption, first we proposed the CRG model in this paper to represent the complex mechanical product. The pruning method of the CRG can identify the subassembly so that many unnecessary components will not be included in the disassembly sequence. The disassembly of the identified subassembly is selective because some groups of the components can be disassembled as an entirety without releasing the inner-connections between them. Second, we established elaborate models for more realistic situations to calculate different kinds of energy consumption during the disassembly process. Third, the pheromone factor is proposed to improve the performance of ACO and the proposed IACO is used to deal with the variable number of the components included in the final disassembly sequence.

By combining the CRG and IACO, the energy consumption of the disassembly to recycle the target component is minimized. The integrated method is applied to a rolling machine for recycling a target component and the comparisons with other heuristic algorithm showed that the proposed method is effective. The results of the experimental simulation also indicate that the energy consumption in different aspects during the disassembly process is saved. In a word, the work in this paper provides a novel and efficient way to recycle a target component from a complex mechanical product regarding energy-savings.

One limitation of the proposed approach is that the parameters have a great influence on the performance of the algorithm such that many test experiments need to be conducted to determine the appropriate values. The sensitivity analysis of the parameters can be a direction of the future research. Another limitation is that the stochastic factors such as the skill level of workers and unexpected machine breakdown in the disassembly process are not considered, which could be another topic for further study.

Author Contributions: All authors have equally contributed to this article.

Funding: This work was funded by the National Natural Science Foundation of China (No. 51775489), Zhejiang Provincial Natural Science Foundation of China (No. LZ18E050001), and the National Key Research and Development Program of China (No. 2017YFB1301201).

Conflicts of Interest: The authors declare no conflict of interest.

\section{Nomenclature}

D The set of directed edge in the CRG

$d_{i, j} \quad$ The element of $D$

$E \quad$ The set of undirected edge in the CRG

$E_{b} \quad$ The total basic equipment energy consumption

$E_{c} \quad$ The total energy consumption of tool changes

$e c_{i, j} \quad$ The total energy consumption removing component $j$ from its adjacent component $i$

$e_{c i} \quad$ The energy consumption of the $i$-th tool change

$E_{d} \quad$ The total energy consumption of disassembly operation direction changes

$e_{d i} \quad$ The energy consumption of the $i$-th disassembly operation direction change

$e_{i, j} \quad$ The element of $E$ 
$E_{k} \quad$ The total energy consumption of $k$-th ant's path

$E_{m} \quad$ The total energy consumption caused by component-removing operations

$e_{m i} \quad$ The energy consumption of the $i$-th component-removing operation

Energy The total energy consumption required to complete the disassembly process

$E_{r} \quad$ The total energy consumption caused by releasing the inter-connections

$e_{r i} \quad$ The energy consumption of releasing the $i$-th inter-connection

$E_{S} \quad$ The energy consumed by support work

$E_{v} \quad$ The energy consumption vector

G The CRG model

The max generation of the improved ant colony algorithm

$M \quad$ The number of the equipment

$m \quad$ The number of ants in colony

$n \quad$ the number of components to be disassembled

$n_{d} \quad$ The number of the components included in the subassembly

$n_{k}$

$P_{b i}$

The number of components in the disassembly sequence represented by the $k$-th ant's path

The average power of the basic equipment energy consumption

$P_{k} \quad$ A point set in which all the points are allowed to visit by the ants

$P_{S} \quad$ An ordered point set that records the disassembly sequence

$P_{i, j}^{k} \quad$ The probability that the $k$-th ant will transfer from point $i$ to point $j$

$t_{b i} \quad$ The total preparing time of the $i$-th equipment

$Q \quad$ The total pheromone released by one ant

$T_{c} \quad$ The time of tool changes

$T_{d} \quad$ The time of disassembly operation direction changes

$T_{m} \quad$ The number of component-removing operations

$T_{r} \quad$ The number of releasing operations

$V \quad$ The point set of the CRG

$v_{i} \quad$ The element of $V$

$W \quad$ The weight set of the CRG

$w_{i, j} \quad$ The element of $W$

$\alpha \quad$ The importance degree factor of pheromone

$\beta \quad$ The importance degree factor of heuristic function

$\rho \quad$ The volatilization factor of pheromones

$\eta_{i, j} \quad$ The heuristic function

$\Delta \tau_{i, j} \quad$ The sum amount of pheromones that all the ants left between the nodes $i$ and $j$

$\Delta \tau_{i, j}^{k} \quad$ The amount of pheromone that the $k$-th ant left between nodes $i$ and $j$

\section{References}

1. Haapala, K.R.; Zhao, F.; Camelio, J.; John, W.S.; Steven, J.S.; David, A.D.; Jawahir, I.S.; Andres, F.C.; Jeremy, L.R. A review of engineering research in sustainable manufacturing. ASME J. Manuf. Sci. Eng. 2013, 135, 599-619. [CrossRef]

2. Wu, Y.; Saxena, S.; Xing, Y.; Wang, Y.; Li, C.; Yung, W.K.; Pecht, M. Analysis of Manufacturing-Induced Defects and Structural Deformations in Lithium-Ion Batteries using Computed Tomography. Energies 2018, 11, 925. [CrossRef]

3. Xia, K.; Gao, L.; Wang, L.; Li, W.; Chao, K. A semantic information services framework for sustainable WEEE management toward cloud-based remanufacturing. ASME J. Manuf. Sci. Eng. 2015, 137, 235-257. [CrossRef]

4. Guiras, Z.; Turki, S.; Rezg, N.; Dolgui, A. Optimization of two-level disassembly/remanufacturing/assembly system with an integrated maintenance strategy. Appl. Sci. 2018, 8, 5. [CrossRef]

5. $\quad$ Feng, Y.; Zhou, M.; Tian, G.; Li, Z.; Zhang, Z.; Zhang, Q.; Tan, J. Target disassembly sequencing and scheme evaluation for CNC machine tools using improved multiobjective ant colony algorithm and fuzzy integral. IEEE Trans. Syst. Man Cybern. Syst. 2018, 1-14. [CrossRef]

6. Feng, Y.; Gao, Y.; Tian, G.; Li, Z.; Hu, H.; Zheng, H. Flexible process planning and end-of-life decision-making for product recovery optimization based on hybrid disassembly. IEEE Trans. Autom. Sci. Eng. 2018, 1-16. [CrossRef] 
7. Gaustad, G.; Olivetti, E.; Kirchain, R. Design for recycling: Evaluation and efficient alloy modification. J. Ind. Ecol. 2010, 14, 286-308. [CrossRef]

8. Cerdas, F.; Titscher, P.; Bognar, N.; Schmuch, R.; Winter, M.; Kwade, A.; Herrmann, C. Exploring the Effect of Increased Energy Density on the Environmental Impacts of Traction Batteries: A Comparison of Energy Optimized Lithium-Ion and Lithium-Sulfur Batteries for Mobility Applications. Energies 2018, 11, 150. [CrossRef]

9. Tian, G.; Zhang, H.; Feng, Y.; Wang, D.; Peng, Y.; Jia, H. Green decoration materials selection under interior environment characteristics: A grey-correlation based hybrid MCDM method. Renew. Sustain. Energy Rev. 2018, 81, 682-692. [CrossRef]

10. Zhang, Z.F.; Feng, Y.X.; Tan, J.R.; Jia, W.Q.; Yi, G.D. A novel approach for parallel disassembly design based on a hybrid fuzzy-time model. J. Zhejiang Univ. Sci. A 2015, 16, 724-736. [CrossRef]

11. Aydemir-Karadag, A.; Turkbey, O. Multi-objective optimization of stochastic disassembly line balancing with station paralleling. Comput. Ind. Eng. 2013, 65, 413-425. [CrossRef]

12. Zhou, M.C.; Venkatesh, K. Modeling, Simulation, and Control of Flexible Manufacturing Systems: A Petri Net Approach; World Scientific Publishing Co. Pte. Ltd.: Singapore, 1998.

13. Guo, X.; Liu, S.; Zhou, M.; Tian, G. Disassembly Sequence Optimization for Large-Scale Products with Multiresource Constraints using Scatter Search and Petri Nets. IEEE Trans. Cybern. 2016, 46, 2435-2446. [CrossRef] [PubMed]

14. Gao, M.; Zhou, M.; Huang, X.; Wu, Z. Fuzzy Reasoning Petri Nets. IEEE Trans. Syst. Man Cybern. Syst. 2003, 33, 314-324. [CrossRef]

15. Li, J.R.; Khoo, L.P.; Tor, S.B. An object-oriented intelligent disassembly sequence planner for maintenance. Comput. Ind. 2005, 56, 699-718. [CrossRef]

16. Hui, W.; Dong, X.; Duan, G.H. A genetic algorithm for product disassembly sequence planning. Neurocomputing 2008, 71, 2720-2726. [CrossRef]

17. Luo, Y.; Peng, Q.; Gu, P. Integrated multi-layer representation and ant colony search for product selective disassembly planning. Comput. Ind. 2016, 75, 13-26. [CrossRef]

18. Ilgin, M.A.; Taşoğlu, G.T. Simultaneous determination of disassembly sequence and disassembly-to-order decisions using simulation optimization. J. Manuf. Sci. Eng. ASME 2016, 138. [CrossRef]

19. Go, T.F.; Wahab, D.A.; Rahman, M.A.; Ramli, R.; Hussain, A. Genetically optimised disassembly sequence for automotive component reuse. Expert Syst. Appl. 2012, 39, 5409-5417. [CrossRef]

20. McGovern, S.M.; Gupta, S.M. A balancing method and genetic algorithm for disassembly line balancing. Eur. J. Oper. Res. 2007, 179, 692-708. [CrossRef]

21. Wang, J.F.; Liu, J.H.; Li, S.Q.; Zhong, Y.F. Intelligent Selective Disassembly Using The Ant Colony Algorithm. AI EDAM 2003, 17, 325-333. [CrossRef]

22. Ding, L.P.; Feng, Y.X.; Tan, J.R.; Gao, Y.C. A new multi-objective ant colony algorithm for solving the disassembly line balancing problem. Int. J. Adv. Manuf. Technol. 2010, 48, 761-771. [CrossRef]

23. Shan, H.; Li, S.; Huang, J.; Gao, Z.; Li, W. Ant colony optimization algorithm-based disassembly sequence planning. In Proceedings of the 2007 International Conference on Mechatronics and Automation, Harbin, China, 5-8 August 2007; pp. 867-872.

24. Li, W.D.; Xia, K.; Gao, L.; Chao, K.M. Selective disassembly planning for waste electrical and electronic equipment with case studies on liquid crystaldisplays. Robot. Comput. Intergr. Manuf. 2013, 29, 248-260. [CrossRef]

25. Kalayci, C.B.; Gupta, S.M. A particle swarm optimization algorithm with neighborhood-based mutation for sequence-dependent disassembly line balancing problem. Int. J. Adv. Manuf. Technol. 2013, 69, 197-209. [CrossRef]

26. Gao, Y.; Wang, Q.; Feng, Y.; Zheng, H.; Zheng, B.; Tan, J. An Energy-Saving Optimization Method of Dynamic Scheduling for Disassembly Line. Energies 2018, 11, 1261. [CrossRef]

27. Kalayci, C.B.; Hancilar, A.; Gungor, A.; Gupta, S.M. Multi-objective fuzzy disassembly line balancing using a hybrid discrete artificial bee colony algorithm. J. Manuf. Syst. 2015, 37, 672-682. [CrossRef]

28. Gao, Y.; Feng, Y.; Zhang, Z.; Tan, J. An optimal dynamic interval preventive maintenance scheduling for series systems. Reliab. Eng. Syst. Safe 2015, 142, 19-30. [CrossRef]

29. Srinivasan, H.; Figueroa, R.; Gadh, R. Selective disassembly for virtual prototyping as applied to de-manufacturing. Robot. Comput. Intergr. Manuf. 1999, 15, 231-245. [CrossRef] 
30. Smith, S.S.; Chen, W.H. Rule-based recursive selective disassembly sequence planning for green design. Adv. Eng. Inform. 2011, 25, 77-87. [CrossRef]

31. Wilson, R.J.; Watkins, J.J. Graphs: An Introductory Approach: A First Course in Discrete Mathematics; John Wiley \& Sons: Hoboken, NJ, USA, 1990.

32. Wang, K.; Li, H.; Feng, Y.; Tian, G. Big data analytics for system stability evaluation strategy in the energy internet. IEEE Trans. Ind. Inform. 2017, 13, 1969-1978. [CrossRef]

33. Feng, Y.; Hu, B.; Hao, H.; Gao, Y.; Li, Z.; Tan, J. Design of distributed cyber-physical systems for connected and automated vehicles with implementing methodologies. IEEE Trans. Ind. Inform. 2018. [CrossRef]

34. Suga, T.; Saneshige, K.; Fujimoto, J. Quantitative disassembly evaluation. In Proceedings of the 1996 IEEE International Symposium on Electronics and the Environment (ISEE), Dallas, TX, USA, 6-8 May 1996; pp. 19-24.

35. Zhang, X.F. Complex Product Disassembly Analysis and Structure Design for Low-Carbon; Zhejiang University: Hangzhou, China, 2011.

(C) 2018 by the authors. Licensee MDPI, Basel, Switzerland. This article is an open access article distributed under the terms and conditions of the Creative Commons Attribution (CC BY) license (http:// creativecommons.org/licenses/by/4.0/). 